\title{
Complete genome assembly and characterization of an outbreak strain of the causative agent of swine erysipelas - Erysipelothrix rhusiopathiae SY1027
}

\author{
Amy HY Kwok ${ }^{1 \dagger}$, Yufeng $\mathrm{Li}^{2+}$, Jingwei Jiang ${ }^{1}$, Ping Jiang ${ }^{2}$ and Frederick C Leung ${ }^{1,3^{*}}$
}

\begin{abstract}
Background: Erysipelothrix rhusiopathiae is the causative agent of animal erysipelas and, to a fewer occurrences, human erysipeloid. It is ubiquitous in nature and commensal in diverse species of animals, wild or domestic, from mammals and birds to reptiles and fish. Mechanisms of its virulence and pathogenicity are poorly understood.

Results: Making use of the complete genome sequencing of E. rhusiopathiae strain SY1027 and comparative genome analysis between the three highly pathogenic strains (SY1027, Fujisawa and ATCC19414), the genomic structure and putative functional elements, such as pathogenicity island (PAI)-like regions, potential virulence factors and horizontal transferring genes of the bacteria are identified. Strain SY1027 genome is 1,752,910 base pairs long, just 30 kilobases smaller than strain Fujisawa, with the same GC level of 36.36\%. It contains 1,845 open reading frames (ORF) predicted by GLIMMER 3.02, of which 1,775 were annotated by PGAAP, 1,757 ( 95.23\%) were annotated by NCBI nr blast, 1,209 by COG database and 1,076 by KEGG database. 37 potential virulence factors were annotated in strain SY1027 by VFDB, while $19(\sim 51.35 \%)$ of them are common in the 2 strains, 7 of which are potentially related to antibiotic resistance and highly conserved ( $\sim 98-100 \%$ match identity (ID)) amongst the three strains of E. rhusiopathiae and modestly homologous to other gastrointestinal tract-inhabiting Firmicutes ( 40\% match ID), e.g. Clostridium spp., Enterococcus spp. Genomic island- and pathogenicity island-like regions were also predicted, in which some showed association with tRNA and potential virulence factors.
\end{abstract}

Conclusion: Complete genome sequencing of Erysipelothrix rhusiopathiae, the causative agent of animal erysipelas, was performed. Molecular identification of various genomic elements pave the way to the better understanding of mechanisms underlying metabolic capabilities, pathogenicity of swine erysipelas and prospective vaccine targets besides the widely used SpaA antigens.

Keywords: Erysipelothrix rhusiopathiae, Complete genome assembly, Genome characterization, Erysipelas, Virulence factors

\section{Background}

Since its first isolation from mice in 1876, efforts have been devoted to the studies of Erysipelothrix rhusiopathiae (see review: [1]). E. rhusiopathiae is a Gram-positive bacterium that has diverse cell morphology, remarkable chemical tolerance and poorly understood cellular structure and molecular mechanisms, especially those related

\footnotetext{
* Correspondence: fcleung.njau@gmail.com

${ }^{\dagger}$ Equal contributors

'Bioinformatics Center, Nanjing Agricultural University, F202, South Block, Faculty of Science Complex, 1 Weigang, Nanjing 210095, China

${ }^{3}$ School of Biological Sciences, University of Hong Kong, Hong Kong, SAR, China

Full list of author information is available at the end of the article
}

to its virulence in a wide range of hosts, ranging from mammals and birds to reptiles and fish. Best known as the etiological agent of swine erysipelas- a disease that can cause acute symptoms such as septicaemia or lead to chronic syndromes like arthritis and endocarditis in pigs [1], E. rhusiopathiae is generally believed to be transmitted via the gastrointestinal (GI) tract by intake of contaminated water or food and causes great economical loss in husbandry worldwide. Given its ubiquitous nature in the environment and reservoir of asymptomatic carriers amongst both domestic and wild animals, the prevention and control of swine erysipelas is often challenging. More than 23 serovars have so far been described [2]. However, due to the considerable variance in their morphology, host 
specificity and/or pathogenicity, the practicality of serotyping remains debatable. The heat labile capsule of E. rhusiopathiae has been associated with its resistance to phagocytosis and virulence by transposon mutagenesis study [3]. Surface proteins such as neuraminidase, SpaA antigen, two adhesive surface proteins containing the C-terminal anchoring LPXTG motif (RspA and RspB) have shown positive correlation to virulence of the bacteria [4-6], however, little is known about the mechanisms of its pathogenicity.

In China, outbreaks of swine erysipelas have surfaced in recent years despite its relative tranquility in the past 3 decades, with an alarming trend of developing from scattered, domestic occurrences in a few farms to systemic outbursts in provincial scale. Three representative local reports, which are only available in Chinese, are included in Additional file 1. In present study, we isolated an outbreak field strain SY1027 from SiYang, Jiangsu and completed its bacterial genome sequencing and assembly. The complete genome of E. rhusiopathiae strain Fujisawa has just recently been published [7]. Together with the draft genome of strain ATCC19414, data-mining of potential virulence factors, especially those related to capsular protein biosynthesis, would offer us a more reliable comparative genomic analysis and a better understanding of the common genomic structure and a more precise prediction of potential virulence factors for improvement of vaccine targets or strategy of disease control.

\section{Methods}

\section{Isolation and total DNA extraction of E. rhusiopathiae strain SY1027}

E. rhusiopathiae strain SY1027 was collected from a pig farm in SiYang, Jiangsu. Anticoagulant-treated blood was collected from diseased pigs by in-house veterinarians from pig farms following the Nanjing Agricultural University Animal Ethics Committee guidelines (Approval No. IACECNAU20100902) and sent to our laboratory. The sample was inoculated in Martin's broth supplemented with $400 \mu \mathrm{g} / \mathrm{ml}$ neomycin and $70 \mu \mathrm{g} / \mathrm{ml}$ vancomycin. A single colony was isolated by inoculation of the culture on streaked agar plate supplemented with horse serum.

The single colony of the bacterium was inoculated in Modified Feist Broth (6 g glucose, $5 \mathrm{~g}$ proteose peptone no.2 (BD BioSciences, San Jose, US), 5 g yeast extract, $0.5 \mathrm{~g} \mathrm{~L}$-arginine, $0.5 \mathrm{ml}$ Tween 80 (oleic acid) in $1 \mathrm{~L}$ of $0.2 \mathrm{M}$ sodium phosphate buffer (pH8.0) [8] supplemented with $5 \%(\mathrm{v} / \mathrm{v})$ fetal bovine serum (Invitrogen, Grand Island, US) and $50 \mu \mathrm{g} /$ $\mathrm{ml}$ kanamycin and grown to its exponential growth phase. Bacteria were harvested by centrifuge and its genomic DNA was extracted according to the JGI bacterial DNA isolation CTAB protocol (http://1ofdmq2n8tc36m6i46scovo2e. wpengine.netdna-cdn.com/wp-content/uploads/2014/02/JGIBacterial-DNA-isolation-CTAB-Protocol-2012.pdf).

\section{Pyrosequencing and complete genome assembly of E. rhusiopathiae strain SY1027}

To confirm the purity of the genomic DNA of E. rhusiopathiae strain SY1027, 16S rDNA-specific region was amplified and 20 individual positive clones were sequenced by Genetic Analyzer 3130 (Invitrogen, Grand Island, US). BLASTn analysis [9] revealed that E. rhusiopathiae strain SY1027 gDNA sequences have high similarity to those from the other 2 strains of E. rhusiopathiae publicly accessible. The quality and quantity of genomic DNA were evaluated by $0.7 \%$ agarose gel electrophoresis and Nanodrop 2000 (Thermo Scientific, Waltham, US), and using the Quant-iT Picogreen dsDNA kit (Invitrogen), respectively.

A whole genome shotgun library was generated with $500 \mathrm{ng}$ of $E$. rhusiopathiae strain SY1027genomic DNA. The shotgun sequencing procedure was performed using 454 GS Junior General Library Preparation Kit, following the manufacturer's instruction (Roche, Basel, Switzerland). In addition, an $8 \mathrm{~kb}$ span paired end library was generated with $15 \mu \mathrm{g}$ of $E$. rhusiopathiae strain SY1027 genomic DNA. The paired end sequencing procedure was performed using 454 GS Junior Paired end Library Preparation Kit, following the manufacturer's instruction (Roche). Paired end reads were used as orientation guide for assembling the contigs into scaffolds. The DNA libraries were amplified by emPCR and sequenced by FLX Titanium sequencing chemistry (Roche). One shotgun run and one paired end run were performed on individual libraries prepared with same genomic DNA sample. After sequencing, the raw data were assembled by Newbler 2.7 (Roche) with default parameters. Primer pairs were designed along the sequences flanking the gap regions for PCR gap filling. The complete genome of E. rhusiopathiae strain SY1027 was submitted to NCBI Genbank and is publicly accessible [GenBank: CP005079].

\section{Genome annotation of $E$. rhusiopathiae strain SY1027}

Glimmer 3.02 [10] was used for gene prediction in E. rhusiopathiae strain SY1027 complete genome. All predicted ORF sequences were translated into amino acid sequences by in-house Perl scripts. BLASTp [9] was applied to align the amino acid sequences against the NCBI non-redundant (nr) database (January, 2013). Amino acid sequences with alignment length over $90 \%$ of its own length and over $40 \%$ match identity were chosen and the description of the best hit (with highest alignment length percentage and match identity) was assigned as the annotation of predicted gene. Intergenic regions were annotated by RepeatMasker (http:// www.repeatmasker.org) with default parameters. For fair comparison, genome annotation was simultaneously conducted using PGAAP (NCBI). 


\section{Functional gene analysis}

BLASTp [9] was applied to align the amino acid sequences against the COG database [11]. Amino acid sequences with alignment length over $90 \%$ of its own length and over $20 \%$ match identity were chosen and the description of the best hit (with highest alignment length percentage and match identity) was assigned as the annotation of predicted gene. All annotated genes were then classified based on their COG classes and compared to that of E. rhusiopathiae strain Fujisawa.

\section{Pathway analysis}

GLIMMER-predicted ORF sequences of E. rhusiopathiae strain SY1027were translated into amino acid sequences by in-house Perl scripts. All sequences were submitted to KEGG database [12] for automatic pathway annotation (http://www.genome.jp/kaas-bin/kaas_main) and then manually downloaded and curated by in-house Perl scripts.

\section{Virulence gene analysis}

BLASTp [9] was applied to align the amino acid sequences against the VFDB database [13]. Amino acid sequences with alignment length over $90 \%$ of its own length and over $20 \%$ match identity were chosen and the description of the best hit (with the highest alignment length percentage and match identity) was assigned as the annotation of predicted gene.

\section{Genomic island (GI) and pathogenicity island (PAI) analyses}

Genomic islands and Pathogenicity islands were annotated using IslandViewer (http://www.pathogenomics.sfu.ca/ islandviewer/about.php) [14] and PAI Finder (https:// www.gem.re.kr/paidb/pai_finder.php? $\mathrm{m}=\mathrm{f}$ ) on PAIDB [15] respectively.

\section{Drug resistant gene analysis}

BLASTp [9] was applied to align the amino acid sequences against the ARDB database [16]. Amino acid sequences with alignment length over $90 \%$ of its own length and over $40 \%$ match identity were chosen and the description of the best hit (with the highest alignment length percentage and match identity) was assigned as the annotation of predicted gene. All annotated genes were designated by the antibiotics to which they render the bacteria resistant and compared to that of E. rhusiopathiae strains SY1027.

\section{Annotation of plasmid/phage/prophage-derived genes using ACLAME database}

BLASTp [9] was applied to align the amino acid sequences against the ACLAME database [17]. Amino acid sequences with alignment length over $90 \%$ of its own length and over $40 \%$ match identity were chosen and the description of the best hit (with highest alignment length percentage and match identity) was assigned as the annotation of predicted gene. All annotated genes were classified according to their corresponding potential horizontal transferring vectors ("virus" or phages in bacteria, plasmid or prophage) and then compared to that identified in strain Fujisawa.

\section{Comparative genomic analysis of E. rhusiopathiae strain SY1027 and strain Fujisawa}

Complete genomes of E. rhusiopathiae strain Fujisawa [GenBank: NC_015601] was downloaded from NCBI Genbank. Orthologous genes were identified by reciprocal BLAT [18] using GLIMMER-predicted E. rhusiopathiae strains SY1027 and Fujisawa genes. Predicted genes of $E$. rhusiopathiae strain SY1027 which are found as single copies and with $90 \%$ minimum alignment length in strain Fujisawa were designated as the core genes.

\section{Results and discussion}

Sequencing and assembly of Erysipelothrix rhusiopathiae strain SY1027 complete genome

Erysipelothrix rhusiopathiae strain SY1027 genome was sequenced and its complete de novo assembly was achieved by one shotgun run and one $8 \mathrm{~kb}$-span paired end run followed by PCR gap filling. A total of 65,982 raw shotgun reads $(26,499,381$ bases) and 65,032 raw paired end reads $(19,179,868$ bases) were generated, $\sim 99.83 \%$ and $\sim 85.72 \%$ of them, respectively, were aligned into 59 contigs and 4 scaffolds, yielding an average sequencing depth of $\sim 23$-fold. Average read lengths for the shotgun run and the paired end run are $\sim 400 \mathrm{bp}$ and $\sim 300 \mathrm{bp}$, respectively. The largest scaffold is $1,733,324$ base pairs (bp) in size and contains 43 large contigs, while that of the N50 contig is 73,030 bp, indicating that the raw assembly is highly continuous. After PCR gap filling by Sanger sequencing, the complete circular genome of E. rhusiopathiae strain SY1027 is $1.7 \mathrm{Mb}$ (i.e. $1,752,910 \mathrm{bp)}$ with $36.36 \%$ GC content, which is just $\sim 30 \mathrm{~kb}$ smaller than and share the same GC content to that in strain Fujisawa (Table 1).

\section{Genome annotation of E. rhusiopathiae strain SY1027}

1,845 open reading frames (ORF) were predicted by GLIMMER version 3.02, and 1,757 of them (i.e. 95.32\%) were annotated by BLASTp search against the NCBI nonredundant (nr) database (Mar., 2013). The full annotation result was attached as Additional file 2. Simultaneous annotation using PGAAP yielded 1,775 CDS (NCBI).Both numbers of annotated CDS were similar to that in strain Fujisawa (Table 1). 10 rRNA and 53 tRNA were identified via PGAAP. Majority of them were arranged as large RNA islands- 3 ribosomal RNA ( $r r n$ ) operons (loci located on nucleotide positions of 623,505 to $626,780 \mathrm{bp}, 1,386,290$ to $1,391,831 \mathrm{bp}$, and $1,747,947$ to $1,752,878 \mathrm{bp}$ respectively) and 5 tRNA islands (located on 703,967 to 
Table 1 Summary of E. rhusiopathiae strains SY1027, Fujisawa and ATCC19414 genomes

\begin{tabular}{|c|c|c|c|c|}
\hline & & \multicolumn{3}{|c|}{ E. rhusiopathiae strains } \\
\hline & & SY1027 & Fujisawa & ATCC 19414 \\
\hline Total genome size (bp) & & 1752910 & 1787941 & $1750000^{\#}$ \\
\hline GC level (\%) & & 36.36 & 36.36 & N.A. \\
\hline Predicted ORF & & 1845 (Glimmer) & 1780 (Genbank) & 1717 (Genbank \\
\hline (method) & & 1775 (PGAAP) & & \\
\hline \multicolumn{5}{|l|}{ Annotated ORF $(\% \wedge)$} \\
\hline Against KEGG DB & & $1076(58.32)$ & $1073(60.28)$ & $1080(62.90)$ \\
\hline Against NCBI nrDB & & $1757(95.23)$ & $1697(95.34)$ & $1645(95.81)$ \\
\hline Against COG DB & & $1209(65.53)$ & $1123(63.09)$ & $1423(82.88)$ \\
\hline \multirow[t]{4}{*}{ Against ACLAME DB } & & $226(12.25)$ & $223(12.53)$ & 335 (19.51) \\
\hline & plasmids & $197(10.68)$ & $187(10.51)$ & $277(16.13)$ \\
\hline & phages & $5(0.27)$ & $10(0.56)$ & $14(0.82)$ \\
\hline & prophages & $24(1.30)$ & $26(1.46)$ & $44(2.56)$ \\
\hline Against VFDB & & $37(2.01)$ & $27(1.52)$ & $68(3.96)$ \\
\hline Against ARDB & & $7(0.38)$ & $7(0.39)$ & $14(0.82)$ \\
\hline Against PAIDB & & $5(0.27)$ & $9(0.51)$ & N.A. \\
\hline \multirow[t]{4}{*}{ Repetitive seq in bp (\%*) } & & $9393(0.54)$ & $11225(0.64)$ & N.A. \\
\hline & Small RNA & $5889(0.34)$ & $8032(0.46)$ & N.A. \\
\hline & Low complexity & $3131(0.18)$ & $2874(0.16)$ & N.A. \\
\hline & Interspersed repeats & $91(0.01)$ & $91(0.01)$ & N.A. \\
\hline
\end{tabular}

Footnotes: "denotes the approximate number according to public database; $\wedge$ denotes number of genes in percentage to total number of predicted open-reading frames by Glimmer 3.02; *denotes length of sequence in percentage to total genome length; DB - abbreviation for database; seq- abbreviation for sequence; N.A. - non-applicable as strain ATCC19414 genome is only a draft assembly.

$705,956 \mathrm{bp}, 740,461$ to $741,131 \mathrm{bp}, 1,099,256$ to $1,099,999 \mathrm{bp}, 1,526,860$ to $1,530,608 \mathrm{bp}$, and $1,680,522$ to $1,681,399$ bp respectively). The $r r n$ operons are arranged in the typical order of $16 \mathrm{~S}, 23 \mathrm{~S}$ and $5 \mathrm{~S}$ rRNA genes, with the exception of $r r n$ operon 2 which is composed of an additional copy of $5 \mathrm{~S}$ rRNA gene (data not shown). In comparison to strain Fujisawa (7 rrn operons), a considerably fewer number of 3 rrn operons are present in strain SY1027 genome (Table 1). The loci of PGAAP-annotated CDS, NCBI $n r$ annotated CDS and various RNA islands were labeled in the circular representation of the SY1027 genome (Figure 1).

\section{Comparative genomic analysis of E. rhusiopathiae} strain SY1027

1,277 core genes ( $56.35 \%$ of pan-genome) were identified in the pan-genome (consisted of 2,266 genes) of the three E. rhusiopathiae strains (Figure 2). 61 genes are shared only between strains SY1027 and Fujisawa but not ATCC19414, and only 12 between strains SY1027 and ATCC19414, whilst a far greater number of 240 genes are shared between strains Fujisawa and ATCC19414. Similarly, a greater number of 478 strain-specific genes were identified in strain SY1027, in comparison to 92 in strain Fujisawa and 106 in strain ATCC19414. These figures imply that the strains Fujisawa and ATCC19414 may be more similar to each other than to our strain SY1027, despite preliminary serotyping results that strain SY1027 is of serotype 1a, same with the other two strains (unpublished data).

Synteny between the two complete genomes of strains SY1027 and Fujisawa is generally conserved, apart from a rearrangement observed at the $\sim 850-875 \mathrm{~kb}$ region on strain SY1027 (which corresponded to $\sim 625-650 \mathrm{~kb}$ region on strain Fujisawa) and an unmatched stretch between the two strains which is marked by a red box in Figure 3 .

As suggested by a previous phylogenetic analysis after completion of strain Fujisawa genome sequencing [7], Erysipelothrix spp. form a distinct secluded group sandwiched between the Firmicutes and Mollicutes. Since neither draft nor complete genomes of closely related species e.g. $E$. tonsillarum and E. inopinata were available on GenBank, while those less closely related ones have already been explored in the previous article, hence phylogenetic tree was not constructed in our present study.

COG annotation and comparative analysis of E. rhusiopathiae strain SY1027 with strains Fujisawa and ATCC19414

$1,209$ ( $65.53 \%)$ of all 1,845 predicted ORF were annotated by BLASTp search against NCBI COG database. The top ten COG classes they were annotated in were $[R]$ 


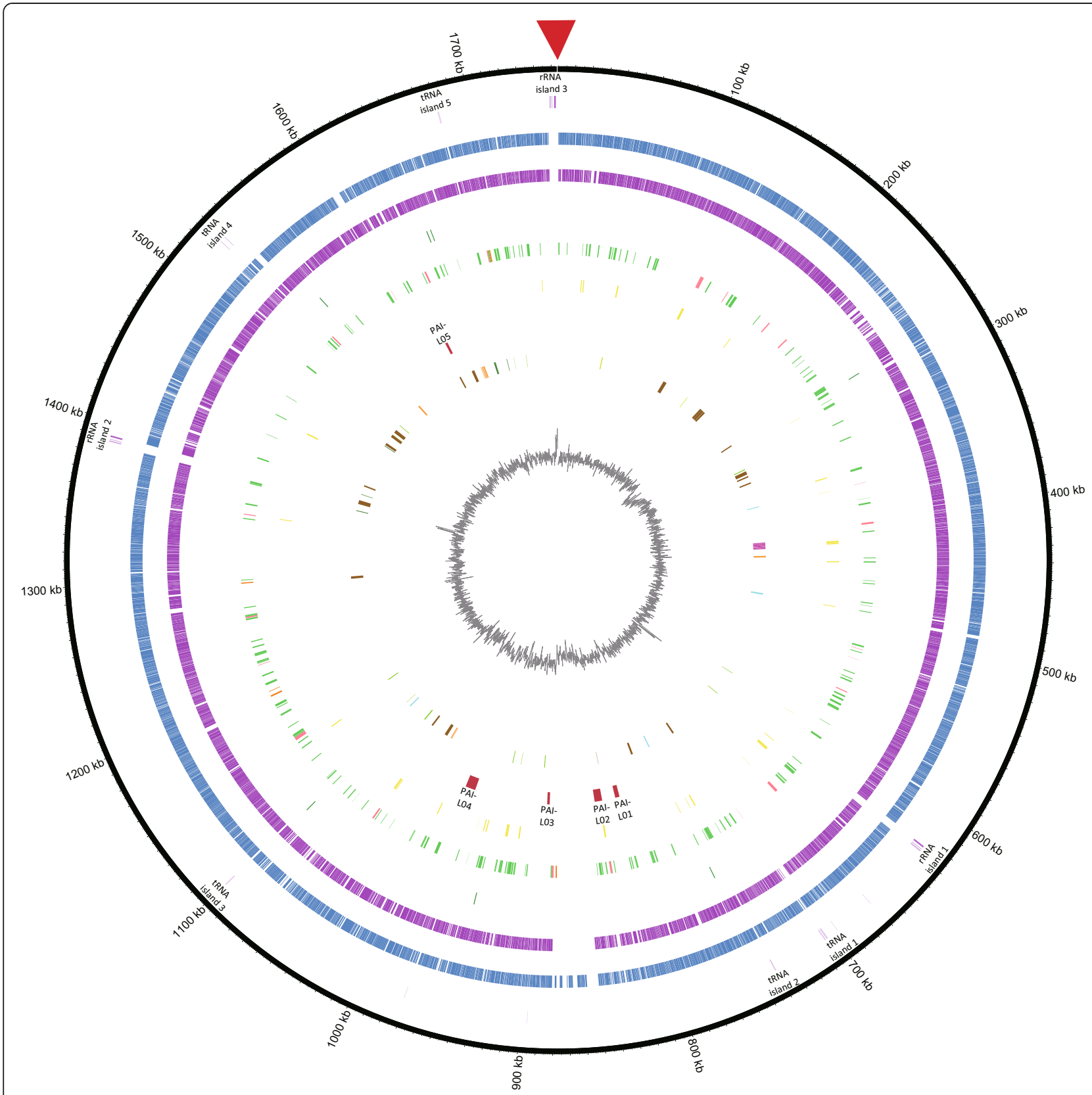

Figure 1 Circular representation of E. rhusiopathiae strain SY1027 genome. From the outer to inner layers, the circle shows (i) nucleotide positions in kilobases (kb) (black); (ii) RNA region whereas rrn operons(light purple) and tRNA islands (dark purple) are labeled accordingly; (iii) PGAAP-annotated CDSs encoded by plus strand (red) and minus strand (dark yellow); (iv) NCBI non-redundant (nr) database-annotated CDSs (blue); (v) genes shared with strain Fujisawa (dark blue); (vi) ACLAME database-annotated potential horizontal transferring genes, classified by their putative origins-plasmid (green), prophage (light red) and phage (orange); (vii) ARDB-annotated potential antibiotics resistance genes (dark red); (viii) percent $\mathrm{G}+\mathrm{C}$ content (gray).

General function prediction only ( 12.08\%), [J] Translation, ribosomal structure and biogenesis $(\sim 11.41 \%),[\mathrm{G}]$ Carbohydrate transport and metabolism ( 9.02\%), [L] Replication, recombination and repair ( 8.52\%), [S] Function unknown ( 7.28\%), [K] Transcription ( 6.20\%), [P] Inorganic ion transport and metabolism $(\sim 5.46 \%),[\mathrm{E}]$
Amino acid transport and metabolism ( 5.29\%), [V] Defense mechanisms ( 4.63\%), and [M] Cell wall/membrane/envelope biogenesis ( 4.30\%). As expected, majority of the genes were involved in basic cellular functions, such as replication, transcription, translation and metabolism, however, up to $\sim 19.36 \%$ of them only have predicted or 


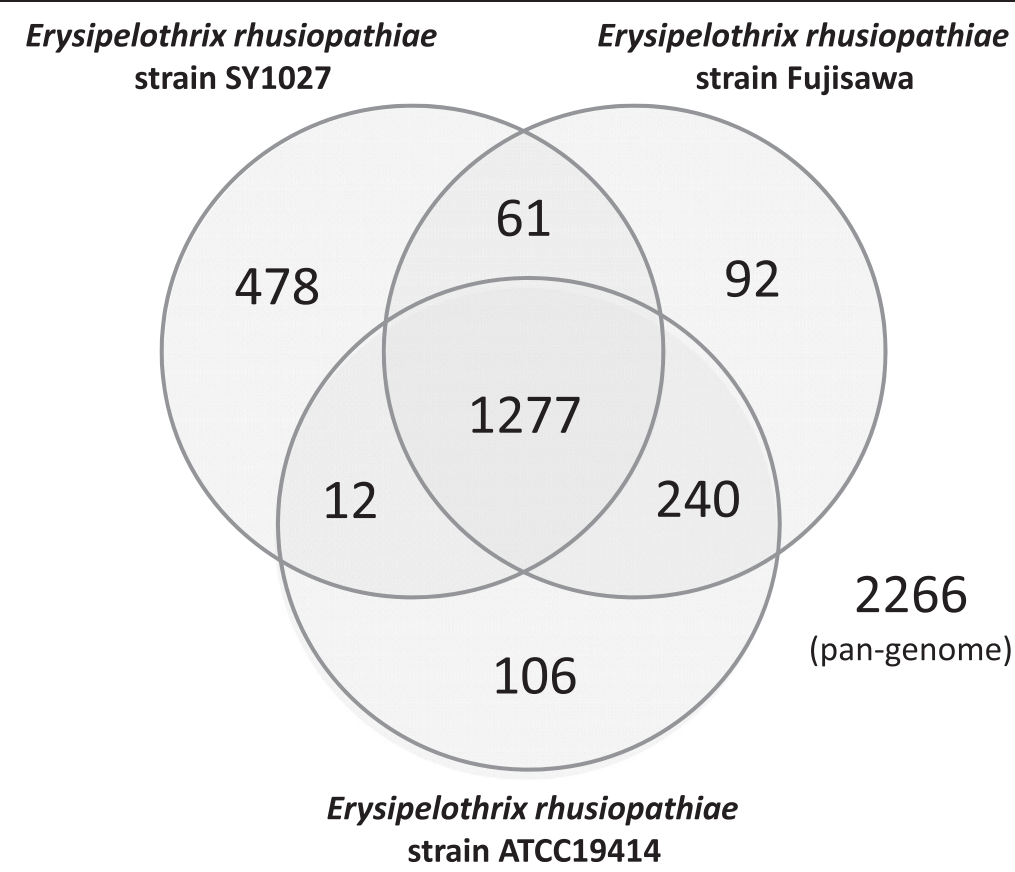

Figure 2 Pan-genome between E. rhusiopathiae strains. This Venn diagram is not drawn in proportion and aims only for the illustration of pan-genome and distribution of common genes including core genes. Circles represent genomes, overlapping regions between circles indicate genes shared with respective genomes. Numeral figures within respective regions denote the number of genes found therein.

unknown functions on COG database. COG class distribution of strain SY1027 was illustrated (Figure 4). Numbers and percentage of each COG class were tabulated as Additional file 3 and their detailed annotations attached as Additional file 4.

Similar numbers of COG-annotated genes were found in strains Fujisawa (1,123 genes; 63.09\%) and ATCC 19414 (1,423 genes; $82.88 \%)$ (Table 1). Percentage of gene annotation in ATCC 19414 is possibly higher due to incomplete sequencing coverage and assembly. COG class distributions are highly similar between strains SY1027 and Fujisawa (Additional files 5 and 6).
Virulence gene/pathogenicity island-like gene annotation and comparative analysis of $E$. rhusiopathiae strain SY1027 and with strains Fujisawa and ATCC19414

37 potential virulence genes $(\sim 2.01 \%$ of total predicted ORF) were identified by BLASTp search against VFDB. Categorized by their respective COG classes, their accession numbers and descriptions are listed in Additional file 7. Many of these potential virulence genes are grouped under or partially involved in cell wall/membrane/envelope biogenesis (COG class $[\mathrm{M}]$ ) or inorganic ion transport and metabolism (COG class $[\mathrm{P}]$ ). The former mainly includes enzymes or proteins involved in capsular polysaccharide

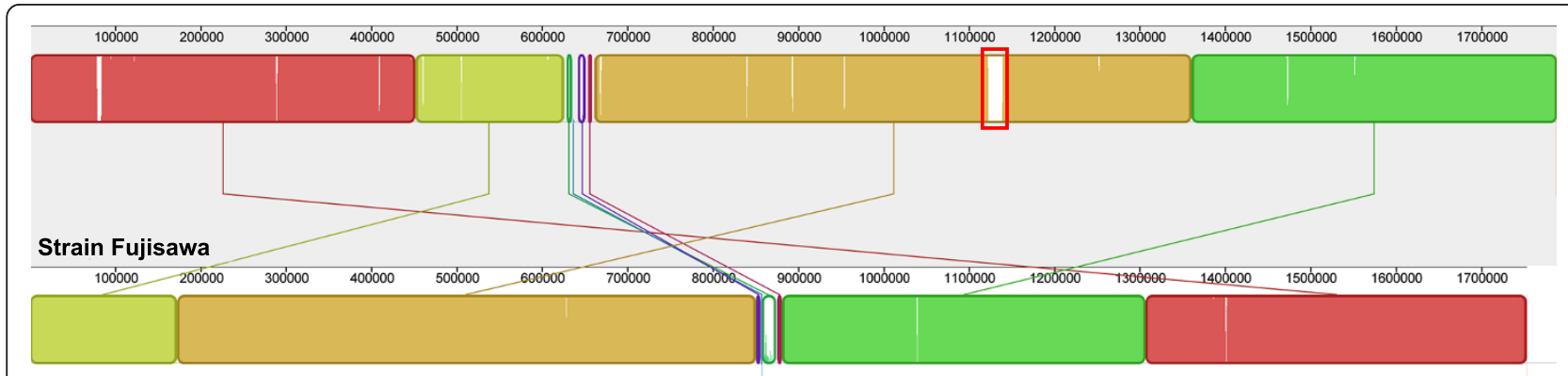

Strain SY1027

Figure 3 Mauve alignment between E. rhusiopathiae strains SY1027 and Fujisawa genomes. The complete genome assemblies are depicted as filled colored blocks in the order they are presented in the respective genomes. The short unmatched stretch found from $\sim 1120$ to $\sim 1140 \mathrm{~kb}$ in Fujisawa genome is marked by a red box. 


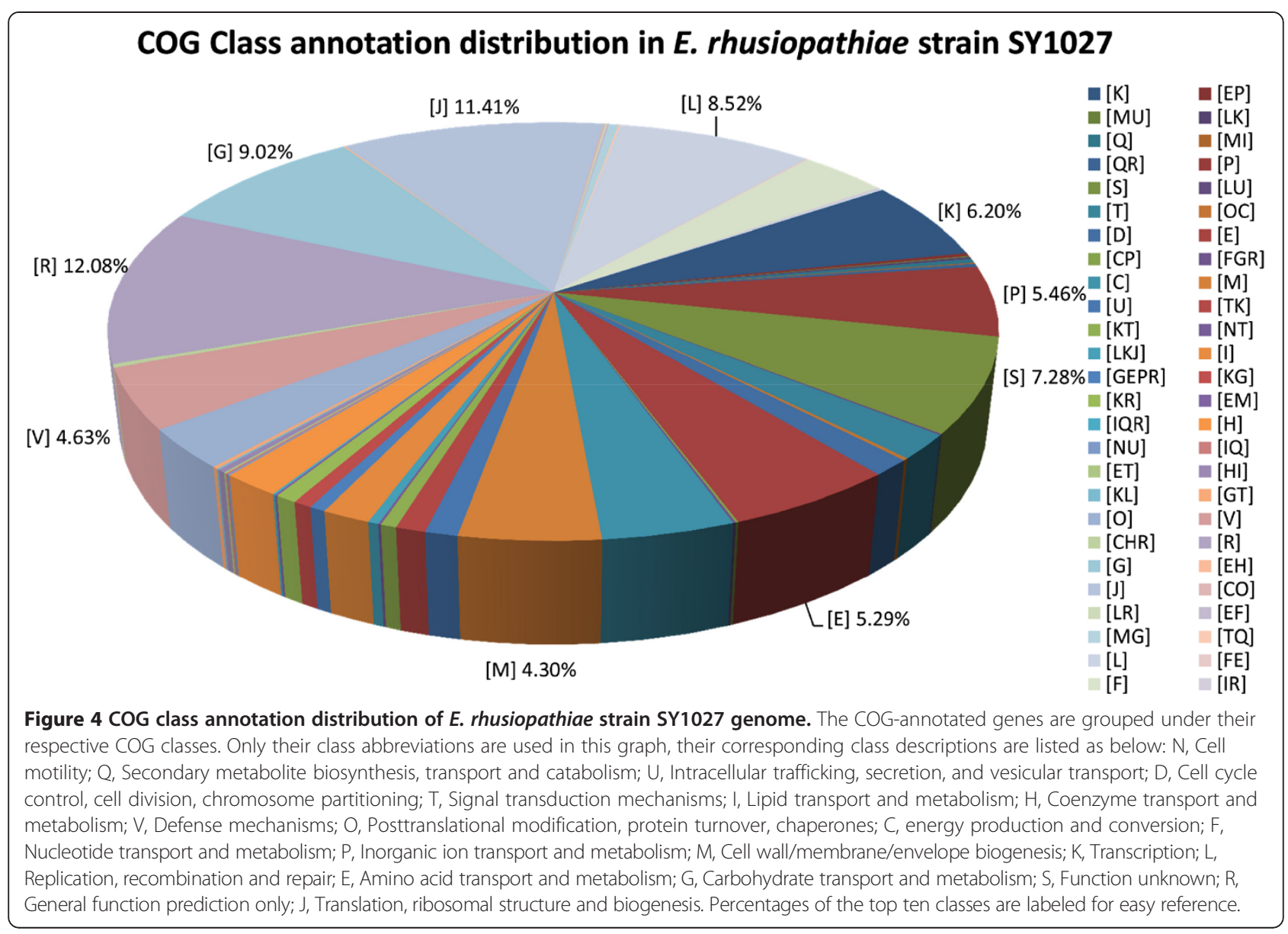

and glycoprotein biosynthesis, and the latter include transporters for iron and magnesium uptake and manganesedependent superoxide dismutase. Metal ions are scarce and limited in biological systems and their uptake by bacteria upon invasion of various host cells have shown positive correlation to bacterial virulence [19-21]. However, their functions and possible relevance to pathogenicity in E. rhusiopathiae remain unresolved. A modestly higher number of virulence genes were identified in strain SY1027 than in strain Fujisawa in VFDB, while the number in strain ATCC19414 might be overestimated due to its draft genome nature (Table 1).

5 genomic island-like regions were annotated via Island Viewer, while 5 pathogenicity island (PAI)-like islands were identified in strain SY1027 via PAI finder in PAIDB (Figure 5, Tables 2 and 3). In comparison to the search result in VFDB, only one PAI-virulence gene (groEL) was annotated in PAI-L02, it may suggest strain SY1027 genome has PAI-like islands atypical of that found in strain Fujisawa and the present entries in the PAIDB. Though GroEL is a chaperonin widely conserved in bacteria, its association to cell adherence and virulence has been described in Clostridium spp. and other bacteria [22,23]. Suggestion of a PAI-island with groEL in our strain may warrant further studies in elucidating its contribution to pathogenicity, as it may offer interesting insights into potential antigenic targets for vaccine development in $E$. rhusiopathiae. In addition to the difference in GC content to the rest of the genome, tRNA were found to flank GI-01, PAI-L03 and PAI-L04, providing further support to these putative genomic islands (Tables 2 and 3). Nevertheless, the virulence of these putative regions in E. rhusiopathiae needs further elucidation, preferably by gene-knockout experiments. Since ATCC19414 genome was only completed to the level of scaffolds, search for GI/PAI-like islands was unfeasible.

Apart from the public databases, a list of potential virulence factors previously suggested in E. rhusiopathiae were identified in the strain SY1027 genome, and they span across various categories, including surface proteins, antioxidant proteins, phospholipases, hemolysins, capsular polysaccharide biosynthesis and extracellular proteins/enzymes (Additional file 8).

Surface proteins were annotated based on their LPXTG motifs, which act as sortase recognition sequence for the covalent binding of their carboxyl termini by sortases in Gram-positive bacteria [4], or GW (glycine-tryptophan) repeats for non-covalent interactions as observed in $E$. 


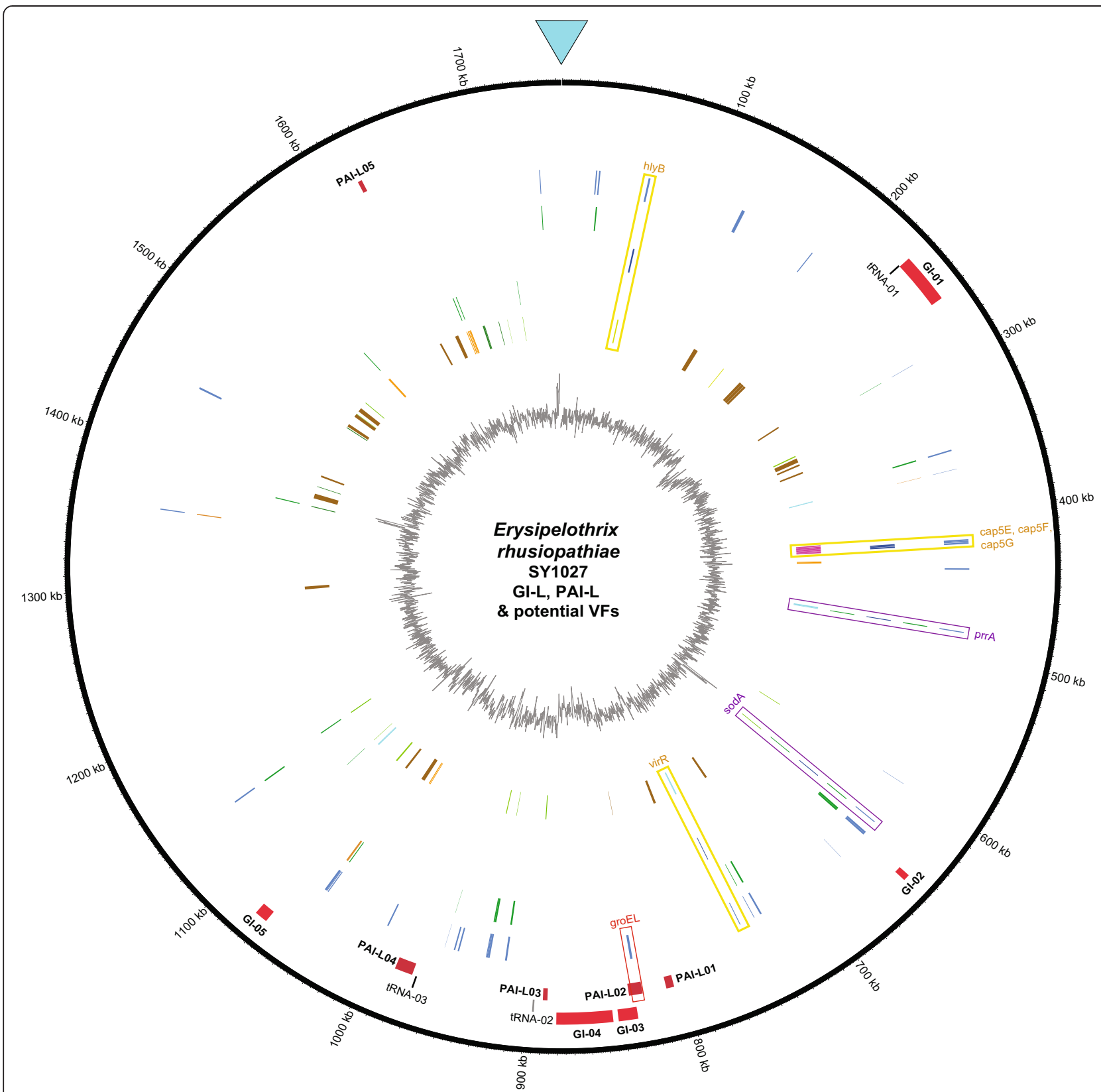

Figure 5 Circular representation of GI-L, PAI-L and potential virulence factors in E. rhusiopathiae strain SY1027 genome. From the outer to inner layers, the circle shows (i) nucleotide positions in kilobases (kb) (black); (ii)Island Viewer-annotated potential genomic islands (Gl) are labeled accordingly (red); (iii) tRNAs flanking the potential Gl or potential PAl-like regions are labeled accordingly, tRNAs found within 5000-bp flanking regions are marked in dark yellow while that found between5000 to 7000-bp flanking region is marked in grey; (iv) PAIDB-annotated PAI-like regions are labeled accordingly (dark red); (v) VFDB-annotated potential virulence factors (blue); (vi) VFDB-annotated potential virulence factors that might have originated from horizontal gene transfer are classified by their putative origins-plasmid (green) and prophage (orange); (vii) VFDB-annotated potential virulence factors that are previously mentioned in the literature are marked in dark green and their positions boxed in yellow and their gene names labeled accordingly. In cases they are predicted to be horizontally transferred from plasmids, they are boxed in purple. (viii) Other potential virulence factors suggested by previous studies that may be horizontally transferred from plasmids (green); (ix) Other potential virulence factors found in the literature suggested by previous studies, divided into 8 categories - two component system with orientation of $5^{\prime}$ histidine kinase-3' response regulator (cyan), two component system with orientation of 3'histidine kinase-5' response regulator (orange), surface proteins (brown), antioxidant proteins (vivid green), phospholipases (avocado green), hemolysins (lime), proteins involved in capsular polysaccharide synthesis (pink) and others (pistachio green); $(x)$ percent $\mathrm{G}+\mathrm{C}$ content (gray). Horizontal transferring genes were annotated by ACLAME database. groEL, VFDB-annotated potential virulence factor that is found within putative PAl-LO2, is boxed in red. 
Table 2 Genomic island-like regions in E. rhusiopathiae strain SY1027 genome

\begin{tabular}{lccccc}
\hline Gl \# & Start & End & Size & \%GC & flanked by tRNA \\
\hline GI-01 & 232197 & 263359 & 31162 & 30.5 & tRNA-Leu (tRNA01) \\
GI-02 & 644770 & 648766 & 3996 & 34.6 & N.F. \\
GI-03 & 831571 & 843384 & 11813 & 32 & N.F. \\
GI-04 & 846580 & 880804 & 34224 & 31.3 & N.F. \\
GI-05 & 1068490 & 1076408 & 7918 & 33.3 & N.F. \\
\hline
\end{tabular}

Footnote: Genomic island-like regions were predicted by IslandPath-DIMOB on IslandViewer (http://www.pathogenomics.sfu.ca/islandviewer/about.php) [14]. N.F.- Not found.

rhusiopathiae [24]. These include SpaA antigen which is commonly used as vaccine target [5], hyaluronidases and neuraminidases which may promote bacterial-host cell surface association [25], RspA and RspB proteins which bind fibronectin and collagen I and II and may participate in biofilm formation [6]. Interestingly, a premature stop codon (TAA) was found near the middle of putative rspA gene (data not shown), leading to the annotation of 2 RspA-like proteins, each representing the N-terminally and C-terminally truncated proteins in strain SY1027 genome (shaded in gray in Additional file 8), instead of 1 in strain Fujisawa. Though our preliminary results suggest that these truncated proteins do not influence the serotyping of strain SY1027 (unpublished data), their conformation, collagen binding capability and influence on hostbacterial interaction may need further elucidation.

E. rhusiopathiae was hypothesized to have an atypical capsule that renders it escape from phagocytosis and antioxidant proteins that facilitates its survival inside polymorphonuclear leukocytes and macrophages [26]. Transposon mutagenesis study showed that the loss of a cluster of genes, putatively a polycistronic mRNA-coding operon, in strain Fujisawa created a mutant deprived of capsule and pathogenicity [3]. The operon was identified, including all 7 proteins potentially involved in capsular polysaccharide biosynthesis, in strain SY1027. Similarly, superoxide dismutase, thioredoxins, thiol peroxidase, alkyl-hydroperoxide reductases and other proteins which confer resistance to oxidative stress, were also found in strain SY1027.
Phospholipases are often considered as potential virulence factors for intracellular pathogens, hypothesized via acquisition of host membrane lipids and disruption of phagosomal membrane. In strain SY1027 genome, 6 phospholipases were identified, same as in strain Fujisawa (Additional file 8).

As E. rhusiopathiae is highly tolerant and almost ubiquitous in nature, two-component regulatory systems which often regulate responses to changes in the environment were also searched for in strain SY1027 genome. 9 pairs of histidine kinase-response regulator were identified- 8 shown similarity to PhoR-PhoB two-component regulatory system and the remaining one to AgrC-AgrA twocomponent regulatory system (Table 4).

The highly potential virulence factors annotated both via VFDB and literature search - hlyB, cap5E, cap5F, cap5G, prrA, sodA and virR (Figure 5) - may require further elucidation on their correlation to pathogenicity, especially in intracellular survival of $E$. rhusiopathiae.

\section{Potential drug resistance gene in E. rhusiopathiae strain SY1027}

7 potential drug resistance genes were annotated in $E$. rhusiopathiae strain SY1027, suggesting it may be resistant to macrolides, vancomycin and teicoplanin (Additional file 9). Their orthologs were also found in strain Fujisawa, sharing $~ 98-100 \%$ match ID (data not shown). Interestingly, a richer reservoir of potential drug resistance genes, both in number and variety, was annotated in strain ATCC19414 (Table 5). Our preliminary antimicrobial results suggested that despite the presence of potential macrolide-resistant genes (which share $~ 40-46 \%$ match IDs to ARDB entries), the antimicrobial susceptibilities to different macrolide-class drugs (e.g. erythromycin, roxithromycin and lincomycin) vary from moderate to minimal levels (unpublished data), hinting that further functional elucidation, preferably gene-knockout experiments, of the potential drug resistance genes are required to substantiate the in silico deduction. In addition, a previous investigation on antimicrobial susceptibilities of E. rhusiopathiae suggested that it has partial or complete resistance to other classes of antibiotics [27] which were not identified

Table 3 Pathogenicity island-like regions in E. rhusiopathiae strain SY1027 genome

\begin{tabular}{|c|c|c|c|c|c|c|c|c|}
\hline PAI-L \# & Start & End & Size & $\% G C$ & flanked by tRNA & PAls homologous to this region ${ }^{\wedge}$ & $\begin{array}{l}\text { No. of ORF in } \\
\text { homo. PAl* }\end{array}$ & $\begin{array}{c}\text { Potential } \\
\mathrm{VF}^{\phi}\end{array}$ \\
\hline PAI-L01 & 806018 & 811037 & 5020 & 37.5 & N.F. & VPI-2 (Vibrio cholerae O1) & 8 & N.F. \\
\hline PAI-L02 & 826289 & 835010 & 8721 & 34.2 & N.F. & $\begin{array}{c}\text { PAI CFT073 (E. coli CFT073), SHI-2 (ShigellaflexneriM90T), } \\
\text { HPI (Yersinia enterocoliticaWA 314) }\end{array}$ & 11 & groEL \\
\hline PAI-L03 & 886571 & 889469 & 2898 & 35.8 & tRNA-Lys (tRNA02) & Not named (Enterococcus faecalis MMH594, V583) & 7 & N.F. \\
\hline PAI-L04 & 973651 & 985197 & 11546 & 36.7 & tRNA-Arg (tRNA03) & Not named (Enterococcus faecalis MMH594, V583) & 15 & N.F. \\
\hline PAI-L05 & 1619897 & 1622633 & 2736 & 36.4 & N.F. & Not named (Enterococcus faecalis MMH594, V583) & 5 & N.F. \\
\hline
\end{tabular}

Footnote: Pathogenicity island-like regions were predicted by PAI Finder (https://www.gem.re.kr/paidb/pai_finder.php?m=f) on PAIDB [15]. ^representative PAls from PAIDB hits; ${ }^{\Phi}$ as annotated by VFDB; ${ }^{*}$ homo. PAI - abbreviation for homologous PAl; N.F.- Not found. 
Table 4 Two-component system genes in E. rhusiopathiae strain SY1027 genome

\begin{tabular}{|c|c|c|c|c|c|}
\hline Contig_orf & Gene & Match ID (\%) & Order & Phylum & COG class and function annotation \\
\hline \multicolumn{6}{|c|}{ Similar to AgrC-AgrA (exoprotein synthesis) two-component regulatory system } \\
\hline contig00001_orf00402 & $\operatorname{lin} 0044$ & 39.42 & Bacillales & Firmicutes & [KT]_COG3279 Response regulator of the LytR/AlgR family \\
\hline contig00001_orf00401 & $\operatorname{lin} 0043$ & 36.19 & Bacillales & Firmicutes & $\begin{array}{l}\text { [1]_COG2972 Predicted signal transduction protein } \\
\text { with a C-terminal ATPase domain }\end{array}$ \\
\hline \multicolumn{6}{|c|}{ Similar to PhoR-PhoB (phosphate starvation response) two-component regulatory system } \\
\hline contig00001_orf00481 & SP2193 & 43.98 & Lactobacillales & Firmicutes & $\begin{array}{l}\text { [TK]_COG0745 Response regulators consisting of a CheY-like } \\
\text { receiver domain and a winged-helix DNA-binding domain }\end{array}$ \\
\hline contig00001_orf00482 & SP0604 & 27.08 & Lactobacillales & Firmicutes & [1]_COG0642 Signal transduction histidine kinase \\
\hline contig00001_orf00536 & CAC2435 & 49.08 & Clostridiales & Firmicutes & $\begin{array}{l}\text { [TK]_COG0745 Response regulators consisting of a CheY-like } \\
\text { receiver domain and a winged-helix DNA-binding domain }\end{array}$ \\
\hline contig00001_orf00535 & CAC2434 & 25.87 & Clostridiales & Firmicutes & [1]_COG0642 Signal transduction histidine kinase \\
\hline contig00001_orf00866 & SP1633 & 52.87 & Lactobacillales & Firmicutes & $\begin{array}{l}\text { [TK]_COG0745 Response regulators consisting of a CheY-like } \\
\text { receiver domain and a winged-helix DNA-binding domain }\end{array}$ \\
\hline contig00001_orf00865 & all3587 & 21.10 & Nostocales & Cyanobacteria & [1]_COG0642 Signal transduction histidine kinase \\
\hline contig00001_orf01180 & $\mathrm{BH} 0372$ & 29.26 & Bacillales & Firmicutes & 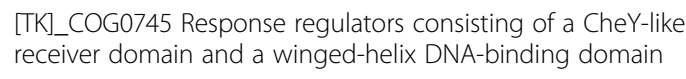 \\
\hline contig00001_orf01181 & BH0754 & 20.07 & Bacillales & Firmicutes & [1]_COG0642 Signal transduction histidine kinase \\
\hline contig00001_orf01262 & CAC0524 & 41.47 & Clostridiales & Firmicutes & 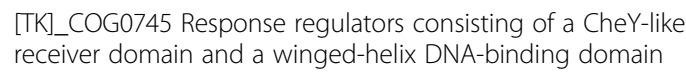 \\
\hline contig00001_orf01261 & Cgl2903 & 24.50 & Actinomycetales & Actinobacteria & [1]_COG0642 Signal transduction histidine kinase \\
\hline contig00001_orf01760 & $\mathrm{BH} 1153$ & 47.39 & Bacillales & Firmicutes & $\begin{array}{l}{[\mathrm{TK}]_{\text {_COG}} \mathrm{CO} 45 \text { Response regulators consisting of a CheY-like }} \\
\text { receiver domain and a winged-helix DNA-binding domain }\end{array}$ \\
\hline contig00001_orf01761 & BS_yrkQ & 27.27 & Bacillales & Firmicutes & [1]_COG0642 Signal transduction histidine kinase \\
\hline contig00001_orf01866^ & CAC0564 & 53.33 & Clostridiales & Firmicutes & $\begin{array}{l}{[\mathrm{TK}] \text { COG0745 Response regulators consisting of a CheY-like }} \\
\text { receiver domain and a winged-helix DNA-binding domain }\end{array}$ \\
\hline contig00001_orf01868 & BS_yvrG & 24.84 & Bacillales & Firmicutes & [1]_COG0642 Signal transduction histidine kinase \\
\hline contig00001_orf01870^ & CAC0830 & 45.02 & Clostridiales & Firmicutes & $\begin{array}{l}{\left[T K{ }_{1} \text { COG0745 Response regulators consisting of a CheY-like }\right.} \\
\text { receiver domain and a winged-helix DNA-binding domain }\end{array}$ \\
\hline contig00001_orf01872^ & BS_yrkQ & 25.19 & Bacillales & Firmicutes & [1]_COG0642 Signal transduction histidine kinase \\
\hline
\end{tabular}

in our present search, hinting the presence of other potential drug resistance genes, either novel or unlisted in the $\mathrm{ARDB}$, in the bacteria.

\section{Potential horizontal transferring elements in E. rhusiopathiae strain SY1027}

Horizontal transferring elements are common in bacteria and generally believed to be a significant driving force in prokaryotic evolution [28]. They are mainly divided into three types based on their respective vectors - plasmids, phages (or viri) and prophages [28]. In strain SY1027 genome, 226 potential horizontal transferring elements $(\sim 12.25 \%$ of all predicted ORFs) were annotated via BLASTp against the ACLAME database (Additional file 10). Amongst them, the majority (197 genes; 87.18\%) were putatively derived from plasmids, while the rest were from phages (5 genes; $2.21 \%$ ) and from prophages (24 genes; 10.61\%). Similar figures were observed in strain Fujisawa genome, with a slightly fewer number of putative plasmid-derived genes and doubled number of putative phage-derived genes. 355 potential horizontal transferring elements were found in the draft genome of strain ATCC19414, yet it might be an over-estimated figure due to incomplete or possible mis-assembly.

Table 5 ARDB-annotated genes in E. rhusiopathiae strains SY1027, Fujisawa and ATCC19414 genomes

\begin{tabular}{lcccccc}
\hline & \multicolumn{5}{c}{ Number of potential resistant genes to... } \\
\cline { 2 - 7 } Species & Macrolide & Vancomycin & Teicoplanin & Lincosamide & Tetracycline & Streptogramin a \\
\hline E. rhusiopathiaeSY1027 & 4 & 2 & 1 & 0 & 0 & 0 \\
E. rhusiopathiae Fujisawa & 4 & 2 & 1 & 0 & 0 & 0 \\
E. rhusiopathiaeATCC19414 & 3 & 2 & 1 & 4 & 2 & 2 \\
\hline
\end{tabular}


As observed in Figure 5, some of the potential virulence factors identified might have originated from horizontal gene transfer. Two of them -prrA and sodA-have high virulence potential owing to their identification via both VFDB and literature search. The former is the transcriptional regulator in a PrrA-PrrB two-component regulatory system related to oxygen control and intracellular replication in Mycobacterium [29], while the latter encodes a superoxide dismutase which may offer a mean of antioxidant defense which is critical for intracellular survival and growth in $E$. rhusiopathiae [26].

\section{KEGG pathway analysis of E. rhusiopathiae strain SY1027}

KEGG pathway analysis was performed for E. rhusiopathiae strain SY1027, and the full list of KEGG pathway annotation was listed in Additional file 11. Strain SY1027 genome contains glucose-specific IIA component in phosphotransferase system (PTS), endoglucanase and glucose6-phosphate isomerase, which suggest that the bacteria is capable in breaking down maltose and trehalose, cellulose and fructose, respectively. The potential of utilizing other sugars besides glucose may partly explain the ubiquitous nature of E. rhusiopathiae in the environment. Similar to strain Fujisawa, many critical genes in the tricarboxylic acid (TCA) cycle were found missing in strain SY1027, with the exception of fumarate hydratase, citrate CoAtransferase, dihydrolipoamidesuccinyltransferase and dihydrolipoamide dehydrogenase. Genome reduction was previously hypothesized in E. rhusiopathiae strain Fujisawa, given the partial loss of fatty acid biosynthesis and DNA repair system similar in the Mollicutes [7]. Likewise, strain SY1027 seems to display these characteristics. Apart from the partial loss of TCA cycle genes, only 34 genes related to DNA repair were identified.

\section{Conclusion}

The complete genome of Erysipelothrix rhusiopathiae strain SY1027 was sequenced and assembled. It is $1,752,910$ base pairs long, just 30 kilobases smaller than strain Fujisawa, with the same GC level of 36.36\%. It contains 1845 open reading frames (ORF) predicted by GLIMMER 3.02, of which 1757 (95.23\%) are annotated by NCBI nr blast, 1209 by COG database and 1076 by KEGG database. 37 potential virulence factors are annotated in strain SY1027 by VFDB, while 19 (51.35\%) of them are common in the 2 strains, 7 of which are potentially related to antibiotic resistance and highly conserved ( 98-100\% match ID) amongst the three strains of E. rhusiopathiae and only modestly homologous to other gastrointestinal tract-inhabiting Firmicutes $(\sim 40 \%$ match ID), e.g. Clostridium spp., Enterococcus spp. Molecular identification of these genomic elements and potential virulence factors offer insights into testing prospective vaccine targets besides the widely used SpaA antigens in swine erysipelas and development of more effective treatment or prevention in control of the disease.

\section{Additional files}

\begin{abstract}
Additional file 1: Local Chinese swine erysipelas outbreak reports.
Three articles (titles shaded in yellow) on local swine erysipelas outbreaks published in Chinese scientific journals.

Additional file 2: NCBI nr database annotation for E. rhusiopathiae strain. The full list of orfs in E. rhusiopathiae strain SY1027 annotated against NCBI non-redundant database.

Additional file 3: COG-annotated genes in E. rhusiopathiae strain SY1027 genome. Tabulated summary of orfs in E. rhusiopathiae strain SY1027 annotated against COG database under various COG classes.

Additional file 4: COG functional annotation for $E$. rhusiopathiae strain SY1027 genome. The full list of orfs in E. rhusiopathiae strain SY1027 annotated against COG database.

Additional file 5: COG class annotation distribution between $E$. rhusiopathiae strains SY1027 and Fujisawa genomes. A bar chart comparing the percentage of each single-letter COG class found between the 2 complete E. rhusiopathiae genomes.
\end{abstract}

Additional file 6: Comparison of COG-annotated genes between E. rhusiopathiae strains SY1027 and Fujisawa. Tabulated comparison between COG-annotated genes under each single-letter COG class from the 2 complete E. rhusiopathiae genomes.

Additional file 7: VFDB-annotated genes between $E$. rhusiopathiae strain SY1027 genome. The list of VFDB-annotated genes in strain SY1027 genome, classified by COG classes. Genes shared between strains SY1027, Fujisawa and ATCC19414 were shaded in light gray while those shared between strains SY1027 and Fujisawa were highlighted in dark gray.

Additional file 8: Other potential virulence factors in $E$. rhusiopathiae strain SY1027 genome. A panel of virulence factors mentioned in the literature was searched in strain SY1027 genome and listed here. ^orf00231, orf00462 and orf00466 are pseudogenes with frame-shift or point mutation(s).

Additional file 9: ARDB-annotated genes in E. rhusiopathiae strain SY1027 genome. List of ARDB-annotated genes and their putative antibiotic resistance.

Additional file 10: ACLAME database-annotated genes in E. rhusiopathiae strain SY1027 genome. Full list of orf annotated via ACLAME database in strain SY1027 genome.

Additional file 11: KEGG database-annotated genes in E. rhusiopathiae strain SY1027 genome. Full list of KEGG database-annotated genes in strain SY1027 genome.

\section{Competing interests}

The authors declare that they have no competing interests.

\section{Authors' contributions}

AHYK prepared the genomic DNA for next-generation sequencing (NGS), post-NGS sequence alignment, genome assembly and annotation and comparative genomic analyses, and the drafting of the manuscript. YL and PJ isolated the bacteria from field sample and participated in design of study and coordination. JJ helped in writing in-house perl script and subsequent bioinformatics analyses. FCL participated in the design of study and coordination. All authors read and approved the final manuscript.

\section{Acknowledgements}

The authors would like to thank Raymond K. H. Hui for handling Roche GS Junior runs for this study. 


\section{Author details}

${ }^{1}$ Bioinformatics Center, Nanjing Agricultural University, F202, South Block, Faculty of Science Complex, 1 Weigang, Nanjing 210095, China. ${ }^{2}$ College of Veterinary Medicine, Nanjing Agricultural University, Room 4031, 4th floor, Shaw Building, 1 Weigang, Nanjing 210095, China. ${ }^{3}$ School of Biological Sciences, University of Hong Kong, Hong Kong, SAR, China.

Received: 1 March 2014 Accepted: 26 June 2014

Published: 2 July 2014

\section{References}

1. Wang Q, Chang BJ, Riley TV: Erysipelothrix rhusiopathiae. Vet Microbiol 2010, 140:405-417.

2. To H, Nagai S: Genetic and antigenic diversity of the surface protective antigen proteins of Erysipelothrix rhusiopathiae. Clin Vaccine Immunol 2007, 14:813-820

3. Shimoji Y, Yokomizo Y, Sekizaki T, Mori Y, Kubo M: Presence of a capsule in Erysipelothrix rhusiopathiae and its relationship to virulence for mice. Infect Immun 1994, 62:2806-2810.

4. Navarre WW, Schneewind O: Surface proteins of gram-positive bacteria and mechanisms of their targeting to the cell wall envelope. Microbiol Mol Biol Rev 1999, 63:174-229.

5. Shimoji Y, Mori Y, Fischetti VA: Immunological characterization of a protective antigen of Erysipelothrix rhusiopathiae: identification of the region responsible for protective immunity. Infect Immun 1999, 67:1646-1651.

6. Shimoji Y, Ogawa Y, Osaki M, Kabeya H, Maruyama S, Mikami T, Sekizaki T: Adhesive surface proteins of Erysipelothrix rhusiopathiae bind to polystyrene, fibronectin, and type I and IV collagens. J Bacterio/ 2003, 185:2739-2748.

7. Ogawa Y, Ooka T, Shi F, Ogura Y, Nakayama K, Hayashi T, Shimoji Y: The genome of Erysipelothrix rhusiopathiae, the causative agent of swine erysipelas, reveals new insights into the evolution of firmicutes and the organism's intracellular adaptations. J Bacterio/ 2011, 193:2959-2971.

8. Groschup MH, Timoney JF: Modified Feist broth as a serum-free alternative for enhanced production of protective antigen of Erysipelothrix rhusiopathiae. J Clin Microbiol 1990, 28:2573-2575.

9. Altschul SF, Madden TL, Schaffer AA, Zhang J, Zhang Z, Miller W, Lipman DJ: Gapped BLAST and PSI-BLAST: a new generation of protein database search programs. Nucleic Acids Res 1997, 25:3389-3402

10. Delcher AL, Bratke KA, Powers EC, Salzberg SL: Identifying bacterial genes and endosymbiont DNA with Glimmer. Bioinformatics 2007, 23:673-679.

11. Tatusov RL, Fedorova ND, Jackson JD, Jacobs AR, Kiryutin B, Koonin EV, Krylov DM, Mazumder R, Mekhedov SL, Nikolskaya AN, et al: The COG database: an updated version includes eukaryotes. BMC Bioinformatics 2003, 4:41.

12. Kanehisa M, Goto S, Sato Y, Furumichi M, Tanabe M: KEGG for integration and interpretation of large-scale molecular data sets. Nucleic Acids Res 2012, 40:D109-D114

13. Chen L, Yang J, Yu J, Yao Z, Sun L, Shen Y, Jin Q: VFDB: a reference database for bacterial virulence factors. Nucleic Acids Res 2005, 33:D325-D328.

14. Langille MG, Brinkman FS: IslandViewer: an integrated interface for computational identification and visualization of genomic islands. Bioinformatics 2009, 25:664-665.

15. Yoon SH, Park YK, Lee S, Choi D, Oh TK, Hur CG, Kim JF: Towards pathogenomics: a web-based resource for pathogenicity islands. Nucleic Acids Res 2007, 35:D395-D400

16. Liu B, Pop M: ARDB-Antibiotic Resistance Genes Database. Nucleic Acids Res 2009, 37:D443-D447

17. Leplae R, Hebrant A, Wodak SJ, Toussaint A: ACLAME: a CLAssification of Mobile genetic Elements. Nucleic Acids Res 2004, 32:D45-D49.

18. Kent WJ: BLAT-the BLAST-like alignment tool. Genome Res 2002, 12:656-664

19. Poyart C, Pellegrini E, Gaillot O, Boumaila C, Baptista M, Trieu-Cuot P: Contribution of Mn-Cofactored Superoxide Dismutase (SodA) to the Virulence of Streptococcus agalactiae. Infect Immun 2001, 69:5098-5106.

20. Smith RL, Kaczmarek MT, Kucharski LM, Maguire ME: Magnesium transport in Salmonella typhimurium: regulation of $\mathrm{mgt} A$ and $\mathrm{mgtCB}$ during invasion of epithelial and macrophage cells. Microbiology 1998, 144:1835-1843.

21. Wyckoff EE, Payne SM: The Vibrio cholerae VctPDGC system transports catechol siderophores and a siderophore-free iron ligand. Mol Microbiol 2011, 81:1446-1458
22. Hennequin C, Porcheray F, Waligora-Dupriet A-J, Collignon A, Barc M-C, Bourlioux P, Karjalainen T: GroEL (Hsp60) of Clostridium difficile is involved in cell adherence. Microbiology 2001, 147:87-96.

23. Zügel U, Kaufmann SHE: Role of heat shock proteins in protection from and pathogenesis of infectious diseases. Clin Microbiol Rev 1999, 12:19-39.

24. Yother J, White JM: Novel surface attachment mechanism of the Streptococcus pneumoniae protein PspA. J Bacteriol 1994, 176:2976-2985.

25. Abrashev I, Orozova P: Erysipelothrix rhusiopathiae neuraminidase and its role in pathogenicity. Z Naturforsch C J Biosci 2006, 61:434-438.

26. Shimoji Y, Yokomizo Y, Mori Y: Intracellular survival and replication of Erysipelothrix rhusiopathiae within murine macrophages: failure of induction of the oxidative burst of macrophages. Infect Immun 1996, 64:1789-1793.

27. Venditti M, Gelfusa V, Tarasi A, Brandimarte C, Serra P: Antimicrobial susceptibilities of Erysipelothrix rhusiopathiae. Antimicrob Agents Chemother 1990, 34:2038-2040.

28. Gogarten JP, Townsend JP: Horizontal gene transfer, genome innovation and evolution. Nat Rev Microbiol 2005, 3:679-687.

29. Ewann F, Jackson M, Pethe K, Cooper A, Mielcarek N, Ensergueix D, Gicquel B, Locht C, Supply P: Transient Requirement of the PrrA-PrrB Two-Component System for Early Intracellular Multiplication of Mycobacterium tuberculosis. Infect Immun 2002, 70:2256-2263.

doi:10.1186/1471-2180-14-176

Cite this article as: Kwok et al:: Complete genome assembly and characterization of an outbreak strain of the causative agent of swine erysipelas Erysipelothrix rhusiopathiae SY1027. BMC Microbiology 2014 14:176.

\section{Submit your next manuscript to BioMed Central and take full advantage of:}

- Convenient online submission

- Thorough peer review

- No space constraints or color figure charges

- Immediate publication on acceptance

- Inclusion in PubMed, CAS, Scopus and Google Scholar

- Research which is freely available for redistribution
C Biomed Central 